\title{
Justificación de los derechos humanos: una mirada desde la metaética y la posibilidad de un enfoque no cognitivista
}

\section{María Fernanda Flores}

Universidad Nacional del Litoral, Argentina

Recibido el 5/5/2019/Aceptado el 11/7/2019

\begin{abstract}
Resumen
El presente artículo tiene como objetivo llevar a cabo una revisión del concepto de agencia en dos propuestas actuales de fundamentación de los derechos humanos, con el fin de mostrar las falencias de la concepción naturalista y sentar las bases para una fundamentación alternativa, desde un enfoque no cognitivista. Para ello consideramos que es preciso determinar en qué medida el concepto de agente retoma la idea naturalista de la atribución de derechos en virtud de los rasgos propiamente humanos. Nuestra hipótesis es que esta interpretación opaca algunos elementos de las teorías contemporáneas que pueden ser dilucidados a la luz de la epistemología moral y la metaética.
\end{abstract}

Justification of human rights: a view from metaethics and the possibility of a non-cognitivist approach

\begin{abstract}
In this paper we aim to carry out a review of the concept of agency in two contemporary accounts of the foundation of human rights, in order to show some defects of the naturalistic approach and also to establish the basis for an alternative ground, from a non-cognitivist approach. Thus, we believe it is necessary to determine to what extent the concept of agent took the naturalistic idea of attributing human rights only by virtue of characterically human features. Our thesis claims that this interpretation turns unclear some elements of the contemporary accounts, which can be better understood by means of moral epistemology and metaethics.
\end{abstract}

Un tópico importante en torno a los derechos humanos que conocemos hoy, refiere a su fundamentación o justificación ya que, para muchos, es una forma de conferirle legitimidad a las versiones expresadas en los distintos documentos internacionales. Uno de los modos de brindar un punto de apoyo es concibiéndolos en un nivel moral
Palabras clave

agencia derechos humanos fundacionismo justificación naturalismo no cognitivismo

\section{Keywords}

agency foundationalism human rights justification naturalism non-cognitivism 
1. Tal es la clasificación, por ejemplo, realizada por Beitz, quien las califica como "naturalistas" (cfr. Beitz, 2009: 49-95). más básico, fundado en las características comunes a todas las personas o, más precisamente, en aquellas que se consideran propiamente humanas. Este tipo de fundamentación data de la modernidad, de la tradición de los derechos naturales, y en la actualidad reaparece bajo otros conceptos como el de agencia, que pone de manifiesto la referencia a los rasgos en virtud de los cuales los individuos están en condiciones de desarrollar plenamente su condición de agentes propositivos. En ocasiones, las teorías que siguen esta línea son interpretadas a la luz de la tradición naturalista, es lo que sucede por ejemplo con las propuestas de Alan Gewirth y James Griffin. ${ }^{1}$ Nuestra tesis es que el naturalismo limita el desarrollo de una justificación normativa adecuada de los derechos humanos. Una de las instancias en que se manifiesta esta dificultad es en el tratamiento del concepto de agente. Éste, consideramos, puede ser sustancialmente revisado a la luz de otras contribuciones de la filosofía moral contemporánea. Por esa razón, procuraremos rearticular dicha noción retomando algunos elementos de la tradición no cognitivista en metaética. A través de este trabajo, nuestra expectativa es realizar una contribución al debate actual sobre la fundamentación de los derechos humanos, no solamente evaluando el potencial de la noción de agencia para brindarles sustento normativo, sino también revisando ese concepto a la luz de los desarrollos que han tenido lugar en el campo de la ética analítica y la epistemología moral contemporánea.

El artículo consta de tres partes. En el primer apartado buscamos esclarecer el carácter de la agencia propiamente humana, atendiendo en particular a las propuestas de Gewirth y Griffin, quienes se sirven de este concepto como base de las justificaciones de los derechos humanos. En la segunda sección, argumentaremos que el naturalismo representa una perspectiva de corto alcance para justificar los derechos humanos sobre la base de la noción de agencia, mediante un análisis más detallado de la estrategia que Gewirth y Griffin usan para fundamentar los derechos humanos. En tercer y último lugar, como ya hemos mencionado, indagaremos la posibilidad de brindar una alternativa de fundamentación no cognitivista, retomando la rama racionalista de esta tradición.

\section{La agencia humana como base en las propuestas fundacionistas}

A lo largo del trabajo referiremos al fundacionismo en su sentido justificatorio, es decir, comprendiendo las teorías que buscan proveer una justificación de los derechos humanos reconocidos institucionalmente, a través de un sustrato normativo todavía más básico que los hace legítimos. Este punto de vista se opone al de determinados enfoques críticos y escépticos que hacen hincapié en el carácter puramente positivo de los derechos humanos rechazando cualquier tipo de fundamentación (por ejemplo, Rabossi, 1989; Rorty, 1993). ${ }^{2}$ Las teorías fundacionistas que abordaremos aquí, en las versiones desarrolladas por Gewirth y Griffin, tienen una estructura en común: parten de ciertas creencias básicas sobre la naturaleza humana y derivan de allí la autoridad de los derechos; para ello ambos autores retoman el concepto de agencia como el sustrato de justificación normativa más básico. Antes de abordar el sentido más específico que ocupa este concepto en sus argumentos, indicaremos algunas características que delimitan su aspecto normativo.

En términos generales, un agente es un ser con la capacidad de actuar y la agencia denota el ejercicio de esta capacidad. Esta acción, en la concepción filosófica más extendida, es interpretada en términos de intencionalidad, es decir, no cualquier acción denota el ejercicio de un agente, sino sólo aquellas movidas por una intención. Además, desde este punto de vista es posible sostener que hay una relación estrecha entre acciones intencionales y razones para la acción, ya sean concebidas como deseos o creencias. Por su parte, algunos autores (por ejemplo, Frankfurt, 1971; Taylor, 1977) 
comenzaron a poner el foco en el carácter distintivo de la agencia humana en contraposición a otros tipos de agentes, retomando una idea que ya aparecía en Kant pero adoptando un punto de vista analítico. ${ }^{3}$ Así, observaron que la voluntad revela (a diferencia de lo que ocurre con los animales no humanos) una estructura racional, determinada por la capacidad de reflexión y deliberación sobre el propio accionar en base a evaluaciones de mérito propias. En consonancia, el sujeto es concebido como un ser con libertad y autonomía, condiciones que por su crucial importancia normativa deberán ser resguardadas. Las propuestas fundacionistas de Gewirth y de Griffin asumen estas ideas, y en base a ello intentan identificar los derechos más básicos de las personas y justificar ciertas características que les atribuimos, como su universalidad, prioridad y obligatoriedad.

Haremos referencia, en primer lugar, al tratamiento de la agencia en la propuesta de Griffin ya que ésta es más familiar respecto a lo que podría denominarse el "modelo de los derechos naturales", como indicaremos luego. Griffin, como mencionamos, pone el acento en las condiciones de existencia distintivamente humanas, destacando la capacidad de formarnos una concepción de nosotros mismos, no sólo en el presente sino también en el pasado y el futuro. Podemos construir, además, una imagen de lo que es una buena vida, y esta puede servir de guía para nuestras elecciones (Griffin, 2008: 32 ss.). A su vez, valoramos estas propiedades y asociamos dicho estatus con nuestra dignidad. El concepto de agencia humana condensa estas ideas y se halla en el núcleo de la teoría de Griffin. Sin embargo, para expresar el concepto en un sentido más sustantivo - recordemos que el fin es derivar y justificar una lista de derechos básicos - Griffin utiliza la expresión personhood, 4 que junto con las consideraciones prácticas (practicalities) constituyen los dos pilares en los que se fundamentan los derechos que reconoce. Técnicamente, personhood denota tres valores: autonomía (la capacidad de escoger un camino propio), provisión mínima (el disponer de acceso a los recursos necesarios para actuar como agentes intencionales) y libertad (la capacidad de actuar para realizar nuestros proyectos sin padecer interferencias de terceros) (Griffin, 2008). En cuanto a las consideraciones prácticas, estas constituyen el segundo fundamento de los derechos y sirven para especificar su contenido, atendiendo a elementos universales de la naturaleza humana (como la naturaleza de la motivación y las necesidades propias de nuestra constitución biológica), así como a información sobre las sociedades particulares (por ejemplo, información sobre las amenazas más comunes contra el ejercicio de la agencia en un contexto determinado y sobre los medios disponibles para su protección)..$^{5}$

De este modo, es claro que para Griffin el rango de agente no indica sólo una condición abstracta: debe poder traducirse en un ejercicio concreto. Cada uno tiene que contar con las posibilidades, recursos y aptitudes básicas para actuar con una perspectiva razonable de alcanzar los resultados deseados. Así, los derechos humanos tienen como fin proteger no sólo a la persona como entidad propositiva, sino también el ejercicio efectivo de esa capacidad. Por el énfasis en la actitud reflexiva y en la capacidad para establecer los medios para alcanzar los fines propuestos, podemos advertir que esta caracterización del individuo está comprometida con una concepción racionalista, en un sentido amplio. Esto implica, como Griffin mismo reconoce, que no todo miembro de la especie humana puede ser considerado agente, pues el modo en que lo define excluye, por ejemplo, a los niños, a personas con algún tipo de discapacidad cognitiva, a individuos en estado de coma, etc. ${ }^{6}$

Veamos ahora el enfoque de Gewirth. Para este filósofo, la característica más básica de las personas es su capacidad de actuar, comprendida en el mismo marco general de la acción intencional que mencionamos anteriormente. Nuevamente, el individuo se define por la cualidad de ser agente. Las condiciones que Gewirth identifica como necesarias para la acción son la voluntad (voluntariness) o libertad, y el propósito
3. Si bien esta concepción de agente que se determina por su propia voluntad ya se halla en Kant, hacemos referencia a estos autores contemporáneos porque interpretan la agencia como una evaluación de segundo orden, es decir, como una reflexión sobre los deseos y motivaciones que constituyen un nivel más básico. Esta interpretación "en niveles" es fundamental en la tradición analítica.

4. Aproximativamente, personhood podría traducirse como "carácter de persona".

5. Las consideraciones prácticas son elementos y características de la naturaleza humana y de las sociedades que sirven para hacer los contenidos de los derechos humanos más determinados. Estas nos proveen información empírica, pues Griffin advierte que el primer fundamento por sí solo nos dice muy poco acerca de los márgenes que deben ser establecidos, y el contenido de los derechos humanos debe ser lo suficientemente determinado para dar lugar a un reclamo efectivo hacia otros; además debe ser adaptable a las distintas condiciones de las sociedades. Esta maleabilidad de los derechos (requirement of social manageability) no hace referencia directa a condiciones de tiempo y espacio particulares sino que se trata de una exigencia conceptual. Sin embargo, esto no impide que los derechos humanos puedan ser aplicados a circunstancias particulares, por ejemplo, el derecho a la libertad de prensa puede ser comprendido como una instancia aplicada del derecho a la libertad de expresión (Griffin 2008: 37 ss.). 6. En relación a ello, el estatus de agente no debe ser comprendido como que aparece en grados. Griffin propone comprender la agencia como un concepto umbral (threshold concept). Así, esta condición determina una clase, en la que se está dentro o no (Griffin, 2008: 44 ss.). Esto, como señaló Rorty (1993), constituye un problema para este tipo de teorías. 
(purposiveness) o intencionalidad. Con el primer elemento refiere al control de la propia conducta y la elección no forzada, bajo el conocimiento de circunstancias relevantes; la segunda propiedad indica cuando el agente se propone determinadas metas, que pueden consistir en la acción misma o en algo que se logra a través de la acción. Cuando esta última condición va acompañada de los medios para una acción exitosa, se la denomina bienestar (well-being), denotando una propiedad que refiere a condiciones sustantivas y habilidades que sirven para realizar los objetivos (Gewirth, 1996: 13). En síntesis, decimos que la libertad y el bienestar son las condiciones necesarias para la acción exitosa. Los derechos, concebidos como garantes de estas condiciones, se derivan a partir de la aplicación de principios formales a través de un método particular (dialectically necessary method), en virtud del cual si yo valoro mi propia agencia debo valorar también la de los demás.

Así, la acción humana provee una base última para la justificación, a juicio de Gewirth, sin cometer petición de principio; el otro elemento fundamental es la racionalidad deductiva que dicta las reglas formales. Al igual que Griffin, Gewirth indica que hay un elemento racional constitutivo en la idea de agencia, por la conciencia o percatamiento (self-awareness) sobre las acciones, ya que afirma que el reconocimiento de los derechos de uno mismo implica reconocer los derechos de los otros agentes y por lo tanto los límites que nos impone hacia los demás en términos de deber. Es así que cada agente es portador de derechos (right-holder) y deberes (duty-bearer) al mismo tiempo.

A la luz del análisis anterior, podemos identificar algunos elementos generales que constituyen el marco de las teorías naturalistas actuales. El primer elemento - y tal vez también el más evidente - es el interés por ciertos atributos distintivamente humanos, como la voluntad, la libertad y la autonomía. Es precisamente esto lo que permite a estas teorías tomar distancia del enfoque causal de la agencia, distinguiendo las acciones humanas del orden de causa y efecto de las cosas. Luego, para definir más esta idea, cabe destacar que no cualquier acción, sólo por el hecho de ser humana, es un acto ejecutado en calidad de agente. Tanto Gewirth como Griffin afirman que el individuo decide o se percata sobre su accionar, y esta propiedad es constitutiva de la agencia e implica restricciones a la hora de determinar quiénes entran efectivamente en la categoría de agentes. Por último, indicamos que en ambos autores el carácter individual de la acción se complementa con referencias al plano práctico, siendo este el que les permite justificar derechos concretos a la luz de condiciones sociales o culturales determinadas.

\section{El naturalismo y la estrategia fundacionista}

En esta sección trataré de mostrar que estas teorías se apartan de la estrategia naturalista clásica de derivar los derechos humanos a partir de lo que se considera el estatus propio de la persona. La idea de la posesión de derechos en virtud de la simple humanidad atraviesa distintas formulaciones de los derechos naturales en la modernidad y es, además, una de las más influyentes del naturalismo; por lo tanto, hay un sentido en el que la herencia de esta tradición es evidente en la obra de los autores objeto de análisis pues, según vimos, la concepción del ser humano como agente refiere a las características comunes a todas las personas sin importar su ubicación, tiempo o estatus social. La cuestión ahora es observar con más detalle cómo se fundamentan los derechos humanos para ver las falencias de la interpretación en la línea de los derechos naturales. Nuestra tesis es que las teorías de las que nos ocupamos exceden significativamente este paradigma, y resultan insuficientes para determinar el alcance de la agencia humana como concepto básico para una justificación adecuada de los derechos humanos. Cuando hablamos de una fundamentación de tipo naturalista, referimos al 
modelo de derechos naturales, afianzado en la modernidad con los aportes de Grocio, Puffendorf y Locke (entre otros), en contraposición a otros tipos de fundamentación, como por ejemplo, las que se basan en acuerdos interculturales, entre las que podemos mencionar la de Walzer (1994) y la de Williams (2006), posturas que Beitz clasifica como teorías del acuerdo (Beitz, 2009). ${ }^{7}$ De cualquier modo, cabe señalar que, estrictamente hablando, no hay algo así como un modelo o paradigma naturalista de los derechos humanos, con elementos estables, aunque es posible determinar algunos rasgos en común que nos permiten distinguir una tradición. Algunas de sus principales características según Beitz son: (i) los derechos humanos son derechos que las personas tendrían incluso en un hipotético "estado de naturaleza", independientemente de las instituciones legales o políticas efectivamente existentes; (ii) son innatos y no pueden ser conculcados; (iii) son universales e inalienables; $y$ (iv) los poseen todas las personas simplemente en virtud de su humanidad.

Si bien Griffin insiste en que la idea de los derechos humanos procedente de la tradición naturalista es incompleta, comparte sin embargo la estructura y el modo de fundamentación que pone en el centro al sujeto. Así, sostiene que estos derechos tienen como finalidad resguardar nuestro estatus como persona humana (human standing). Nuestro carácter de persona y los rasgos que asociamos a ello (autonomía, libertad, racionalidad, etc.) nos permiten derivar una lista convencional de derechos (a la vida, a la seguridad, a la toma de decisiones, la libre expresión, etc.). En este sentido, Griffin indica que estos refieren al agente humano en su desempeño (functioning human agent) y en consonancia afirma que, en lo que concierne al aspecto metafísico, su teoría debe comprenderse como un tipo de naturalismo amplio, que se corresponde en el plano epistemológico con una variación del llamado modelo de la percepción que él mismo describe (Cfr. Griffin, 2008: 111 ss.).

En esta línea, Griffin señala que los derechos humanos se derivan de intereses que deben comprenderse abarcando necesidades humanas naturales en un sentido amplio, esto es, no reducidas al nivel biológico. ${ }^{8}$ Ontológica y epistemológicamente, esto trae aparejado algunos problemas respecto a cuál es el estatus de lo que reconocemos como intereses humanos, de donde surge la lista de valores que luego se expresan como derechos. Al explicar cómo se identifican, Griffin sostiene que hallamos entidades o propiedades que satisfacen las necesidades, incluyendo las más complejas como la autorrealización, y es así como logramos dar con los intereses que eventualmente pueden ser considerados valores. Éstos, además, no son subjetivos sino que nos permiten realizar juicios verdaderos o falsos acerca de la naturaleza humana. ${ }^{9}$ En consonancia, la posición correcta para Griffin es, como mencionamos, un tipo de naturalismo amplio, no reductivo; o en su sentido más literal, expansivo, ya que no limita los hechos naturales a una interpretación estrecha como la positivista, que separa tajantemente la dimensión fáctica de la evaluativa, sino que extiende lo que se comprende como natural a la esfera de los valores. De esta forma, Griffin sostiene que los intereses fundamentales de las personas se determinan mediante la percepción de propiedades no necesariamente fácticas. ${ }^{10}$

La siguiente cuestión que nos interesa del razonamiento de Griffin, porque destaca la relevancia del aspecto social, es cómo los intereses imponen normas de conducta. Por un lado, existen fenómenos (por ejemplo, el dolor) que proveen una justificación sin mediar ningún razonamiento. Pero más importante es una segunda forma en la que la prudencia da lugar a la moralidad, a saber, cuando la norma se expresa en políticas (policies). Una política, por ejemplo 'no matar deliberadamente a un inocente', indica una orden; en conjunto expresan ciertas reglas de las sociedades, no elegidas conscientemente pero tampoco arbitrariamente, sino como resultado de un largo proceso y de experiencias. Explicativamente, el contenido de las políticas puede desarrollarse en base a lo que Griffin llamó consideraciones prácticas.
7. Las teorías del acuerdo son aquellas que conciben los derechos humanos como estándares que son, o pueden llegar a ser, objeto de acuerdo entre miembros de distintas culturas cuyos valores morales y políticos pueden diferir en varios aspectos. Este acuerdo puede expresarse, por ejemplo, como una superposición (overlapping consensus) o núcleo común de doctrinas morales y políticas. Desde esta perspectiva, la autoridad de los derechos humanos positivos se deriva de los valores o códigos morales compartidos (Beitz, 2009: 73 ss.).

8. Entre estas menciona la realización, el disfrute, las relaciones personales, entre otras (cfr. Griffin, 2008: 116).

9. Respecto a la clásica objeción de la falacia naturalista, Griffin señala que su teoría evita caer en ella porque la naturaleza de la cual se derivan los derechos, no se comprende en el sentido estrecho delimitado por las ciencias naturales, que excluye la esfera de los valores (cfr. Griffin, 2008: 35).

10. "We have only a common, extremely vague intuition about the 'natural' or 'empirical' world, one that is full of contentious ontological assumptions. (...) I suggest that our notion of the 'factual' is wide enough to include events of meeting and failing to meet interests. The right position, therefore, is, after all, a kind of naturalism. But not the usual kind of reductive naturalism, in which the boundaries of the 'natural' or the 'factual' are kept relatively tight, that is, roughly in the position that they have long had in the fact/value split in the Hume's view that values cannot be derived from facts." (Griffin, 2008:124). 
11. Respecto a las formas de fundacionismo y los argumentos que lo avalan, seguimos los trabajos de Brink (1989) y Campbell (2015).
12. Este principio es formulado formalmente: "if some predicate $P$ belongs to some subject $S$ because $S$ has a certain quality $\mathrm{Q}$ (where the 'because' is that of sufficient condition), then $\mathrm{P}$ logically must belong to all other subjects S1 to Sn" (Gewirth, 1996: 18).
Gewirth, a diferencia de Griffin, no hace mención explícita a la tradición naturalista, pero vemos que también retoma la idea de los derechos "en virtud de la simple humanidad", apelando al carácter de agente. Más específicamente, para Gewirth los derechos humanos son (i) orientados personalmente, es decir, son requisitos orientados a los individuos en virtud de su propio bien y no desde un punto de vista meramente instrumental; (ii) son necesarios normativamente, esto es, son moralmente obligatorios; y (iii) son requisitos morales, lo cual denota, para Gewirth, tres aspectos distintos pero relacionados: refieren a necesidades, son justificados, e indican exigencias o demandas hacia otros (Gewirth, 1984). De esto se sigue que la base justificatoria de los derechos humanos debe ser un principio moral normativo, que sirva para establecer tales requisitos. Por ello, afirma: "La posesión o existencia de derechos humanos no consiste, en primera instancia, en la posesión de ciertos atributos físicos o mentales, sino en ciertos requerimientos morales justificados..." (Gewirth, 1984: 3). Así, esta clase de derechos son anteriores a cualquier tipo de expresión institucional; su justificación depende de la construcción de un argumento moral montado sobre un análisis de la agencia propositiva.

La estructura de este razonamiento no sólo es fundacionista en el sentido justificatorio en que venimos utilizando esta categoría, sino también en un sentido más estricto en cuanto sostiene que las razones morales justificatorias deben estar basadas en última instancia en un principio moral supremo. ${ }^{11}$ Este principio sustantivo tiene que poder establecer que cada persona debe, moralmente, contar con la protección de los bienes más básicos asociados al ejercicio de su agencia racional. Gewirth afirma que su teoría cumple con esta condición sin incurrir en falacia, porque parte de características generales de la acción y no de un enunciado moral autojustificado o autoevidente, y además por el método que aplica, al que denomina dialécticamente necesario. La premisa inicial de su razonamiento ("Hago X por un fin o propósito F") no nos dice cómo actuar, sino que solamente supone aceptar las condiciones que mencionamos anteriormente (la voluntad y el propósito) y el carácter racional del agente. Así da lugar a una serie de enunciados que cada agente que se reconoce como tal debe aceptar, a riesgo de autocontradicción. Por esta vía arriba al fundamento moral más básico, al que denomina Principio de Consistencia Genérica (PCG), que prescribe que cada agente debe actuar de acuerdo con los derechos genéricos de los demás beneficiarios "como con los suyos propios" (Gewirth, 1984: 17). En otras palabras, cada persona por su propia condición tiene derecho a proteger las condiciones básicas para su accionar, es decir, las que le permiten cumplir sus objetivos y alcanzar el bienestar; y este reconocimiento a su vez trae aparejado, para Gewirth, el reconocimiento y el respeto hacia los derechos de los otros individuos que, como seres racionales, también persiguen su propio bienestar. Así, este principio afirma la igualdad de los derechos de los seres humanos, considerados como agentes actuales o prospectos, y la correlación entre derechos y deberes.

La transición de las normas prudenciales al nivel moral se efectúa por la aplicación del principio de universalizabilidad, que se da por el reconocimiento de otros agentes como iguales: reconocer que los propios derechos nos son atribuidos en calidad de agente, implica reconocer que los demás también cuentan con los mismos derechos y supone un deber correlativo de respetarlos. ${ }^{12}$ Gewirth hace hincapié en la idea de que este reconocimiento mutuo nos permite concebir la sociedad como una comunidad, superando así la antítesis entre los derechos individuales y los deberes atribuidos por la pertenencia a un grupo. De modo que podemos observar que, para Gewirth, son el PCG y el principio de universalizabilidad las bases fundamentales para derivar los derechos. Además, el primero incluye las condiciones propiamente humanas (libertad, bienestar) que también ocupan un lugar fundamental. En este aspecto, el argumento hereda el modo de justificación del modelo de los derechos naturales, pero además introduce una estrategia novedosa por la constricción formal de aceptar deberes correlativos. 
Ante todo esto, debemos considerar entonces si estas teorías son realmente "naturalistas". Creemos que la etiqueta no es incorrecta cuando se propone distinguirlas de otras cuya concepción o modo de justificación están más alejados de la tradición de los derechos naturales, pues la agencia, en sentido normativo, permite interpretar determinados elementos y condiciones (voluntad, libertad, autonomía, etc.) como rasgos esenciales de la vida y la acción humanas, y la comprensión de los derechos como protección de aquellas propiedades efectivamente asume la idea de la atribución de derechos "en virtud de la humanidad". Tal es, por ejemplo, el sentido principal que le da Beitz al realizar la clasificación entre teorías naturalistas y del acuerdo (cfr. Beitz, 2009: 49-95). Sin embargo, teniendo en cuenta los esfuerzos que reflejan las teorías actuales para justificar y derivar legítimamente los derechos sin apelar solamente a un orden moral o estatus dado, creemos que el rótulo del naturalismo puede opacar ciertas consideraciones relevantes, pues consideramos que de este modo se corre el riesgo de dejar en un segundo plano ciertas consideraciones epistemológicas, ontológicas y formales que la ética actual desarrolla y que en buena medida se ven plasmadas en el concepto de agencia.

En síntesis, el punto central es que estas teorías no derivan los derechos humanos de un mero análisis de los rasgos distintivos de la persona humana, sino que combinan los caracteres propios del ser humano "como tal" con diversas consideraciones de otra índole, que no están presentes en la tradición clásica del derecho natural. Entre estas cuestiones podemos mencionar las que tratamos aquí: la preocupación por ofrecer las bases ontológicas y epistemológicas en el naturalismo amplio de Griffin (que además se superpone con la interpretación metaética del naturalismo); o en el caso de Gewirth, es fundamental la aplicación de principios formales y de un método particular, ya que dan lugar a la regla más importante en su teoría, a saber, el PCG; también, en ambos autores es explícito el paso hacia las normas morales, mediante la institución de políticas, como indica Griffin, o por la aplicación del principio de universalizabilidad según Gewirth; además, a diferencia de los derechos naturales que ponen el acento en el individuo como beneficiario de ciertas garantías (que en algunas versiones, como la de Locke, apuntan fundamentalmente a proteger la integridad física, la libertad como interferencia y la propiedad), las teorías fundacionistas actuales que siguen esa línea, sostienen que los derechos deben abarcar un campo mucho más amplio de garantías, y complejizan la relación entre el individuo beneficiario y la sociedad, como se expresa por ejemplo en la teorización de la comunidad y los deberes correlativos que señala Gewirth. Asimismo, la lectura del agente como mero beneficiario pasa por alto el papel que juegan las consideraciones prácticas en la determinación del contenido concreto de los derechos en los argumentos de Griffin.

\section{Hacia una fundamentación no cognitivista de los derechos humanos}

En este último apartado nos proponemos explorar preliminarmente la viabilidad de una fundamentación alternativa, a la que referiremos como no cognitivista. Con esta propuesta, no buscamos realizar una lectura o ampliación de las teorías que vimos hasta aquí, sino brindar otra opción ya que se erige a partir de teorías que en algunos aspectos resultan incompatibles con la perspectiva naturalista. Esto es así porque las fundamentaciones que analizamos anteriormente pueden ser clasificadas como cognitivistas puesto que se apoyan en última instancia en la tesis de que existe una verdadera naturaleza humana pasible de ser analizada y conocida - ya sea que se conciba desde el enfoque del naturalismo amplio de Griffin o según los principios generales de la acción que contiene el PCG enunciado por Gewirth. Las teorías naturalistas tienden a ser clasificadas como realistas y cognitivistas, puesto que sostienen que los juicios morales en los que se basan representan hechos o propiedades objetivas e independientes de la mente (realismo), y son enunciados que representan 
13. Para un análisis más detallado de la relación entre naturalismo, realismo y cognitivismo, cfr. Brink, 1989. En cuanto a la clasificación del naturalismo, como una forma de cognitivismo, cfr. Miller, 2013.

14. En contraposición, por ejemplo, a las teorías del acuerdo (en la clasificación de Beitz, 2009) o a las no fundacionistas (como las de Rabossi, 1989; o Rorty, 1993).

15. Por ejemplo, para Ayer y Stevenson, los juicios morales no difieren de expresiones puramente subjetivas de aprobación o desaprobación como "ihurra!” o “iboo!", en cambio para Hare, si bien expresan un estado conativo, son prescripciones universales y universalizables. hechos o estados del mundo, es decir, son creencias no deseos, preferencias u opiniones (cognitivismo). ${ }^{13}$ El naturalismo busca derivar los derechos de un orden moral o status dado, de modo que el carácter objetivo de los derechos parece radicar, en última instancia, en la representación adecuada de un orden independiente (ya sean valores o propiedades empíricas). Esta corriente, por lo tanto, debe enfrentar ciertas dificultades ontológicas y epistemológicas, puesto que debe dar cuenta del status de las entidades o propiedades que brindan sustento a las normas o juicios morales, y además debe explicar cómo se perciben. No obstante, nos interesa mantener, de las versiones abordadas, la centralidad del sujeto agente y la justificación de los derechos humanos a partir de dicha condición. Consideramos que esta forma de fundamentación tiene la ventaja de que permite sostener la existencia de derechos morales que pueden ser invocados como estándar de crítica de los derechos positivos. ${ }^{14}$ Además, tiene como fin al individuo y no a una clase o colectivo, es decir, se oponen a una concepción instrumental de los derechos, lo cual representa un punto a favor porque nos permite sostener la igual dignidad de las personas y se ajusta mejor a la práctica actual de los derechos humanos.

En principio, pensar una fundamentación no cognitivista puede parecer contradictorio, porque las teorías inscriptas en esta corriente no basan la moral en hechos ni creencias presuntamente objetivas, y por lo tanto no conciben enunciados morales verdaderos sobre los cuales sostener otras razones derivadas. Desde el no cognitivismo, las normas pueden ser comprendidas como expresión de un estado psicológico, aunque esto es interpretado con ciertas variantes según la perspectiva de cada autor. ${ }^{15}$ Sin embargo, la posibilidad de una fundamentación resulta contradictoria sólo si entendemos el fundacionismo en un sentido acotado a la estructura de una teoría basada en verdades morales justificadas; pero en el sentido en que utilizamos el término, no vinculado a la estructura sino a la justificación a través de la apelación a un nivel más básico que la expresión institucional de las normas, no es incompatible. Por otra parte, este cambio de perspectiva no significa renunciar a la pretensión de objetividad, puesto que es posible aspirar a una objetividad distinta a la de la ciencia, como la que se deriva de los postulados de la razón en su uso práctico (Cfr. Darwall et al., 1997: 12; Hare, 1999: 147). Por último, vale aclarar, no cognitivismo no es sinónimo de escepticismo moral, ya que desde una posición no cognitivista se puede recurrir, por ejemplo, a una concepción deflacionaria de verdad.

De este modo, dentro de esta amplia corriente, nos interesa retomar algunas tesis de la rama racionalista, entre las que podemos mencionar el prescriptivismo universal de R.M. Hare (1981; 1999) o el expresivismo normativo de A. Gibbard (1990; 1997). Estas teorías racionalistas se oponen a otras, también pertenecientes al no cognitivismo, que conciben la dimensión conativa (deseos, emociones, sentimientos, etc.) como puramente irracional y que sostienen que bajo ningún aspecto los juicios de la ética son susceptibles de verdad o falsedad; tal es la posición, por ejemplo, del emotivismo de A. J. Ayer (1981) y Ch. Stevenson (1937). La idea que defendemos desde nuestra postura es que las normas son fundamentalmente guías de conducta y expresan el deseo o voluntad de quien los formula, pero también se rigen por reglas y criterios formales, además de que pueden contar (secundariamente) con algún elemento descriptivo en su contenido que les otorgue valor de verdad. En esta tradición, como en la metaética en general, gran parte de los análisis se han desarrollado en torno a la naturaleza del deber, enfocándose en las cualidades del concepto y de los juicios de deber, su vínculo con la acción y la motivación moral, etc. Otros términos que también han tenido un lugar central han sido los de correcto/incorrecto, bueno/malo. Sin embargo, el desarrollo respecto al concepto y la normatividad del derecho, o en torno a la subclase que conforman los derechos humanos, ha sido escaso en esta tradición. Así, lo referente a su normatividad y otras características relacionadas deben ser extraídas, en general, de un examen paralelo al deber o inferidas del análisis de 
las normas en general. Uno de los primeros desafíos que se proyecta es, entonces, distinguir elementos propios del análisis de los derechos y más particularmente de los derechos humanos.

En el tratamiento del deber, que tomamos como punto de partida, desde la visión no cognitivista las normas se justifican mediante razones referidas al agente. Estas no son el tipo de razones morales que exigen adoptar el punto de vista de un 'espectador neutral', aunque precisar qué tipo de razones son demanda adoptar una postura: por ejemplo, para Hare, son hechos puramente fácticos, en cambio para Gibbard, son hechos fácticos pero suponen un elemento propiamente normativo. De cualquier modo, lo que comparten las distintas lecturas es que la relación con la dimensión fáctica no consiste en derivar normas a partir de hechos. Pensando en una justificación de los derechos humanos, algunas de las cuestiones a responder son: ¿qué tipo de razones podemos invocar? y ¿cuál es el status de los hechos? Además, el hecho de que las razones estén referidas al agente no significa que varíen de individuo a individuo porque para la vertiente racionalista del no cognitivismo, los juicios racionales convergen. Aun así, los autores se ven obligados a desplegar distintas estrategias para no caer en una posición relativista.

Desde la visión del expresivismo normativo de Gibbard, el sujeto se concibe como racional, pero la racionalidad no se interpreta como una propiedad predicable de un individuo, sino como expresión en un sistema de normas que lo permite:

Lo principal que debe explicarse no es qué es una 'norma', sino qué es 'aceptar una norma' -o, más precisamente, qué significa que algo esté permitido o sea exigido por 'las normas' que una persona 'acepta'. Yo sostengo que estas nociones son psicológicas: están destinadas a aparecer en una teoría explicativa de la experiencia y acción humana (Gibbard, 1997:182).

Este punto de vista nos permite comprender al agente no sólo como racional en la determinación de sus acciones generales (que como vimos son voluntarias e intencionales), sino también, en la aceptación misma de los derechos y otras normas. Como habían observado Griffin y Gewirth, aunque cada uno desde su propio enfoque, la racionalidad es parte constitutiva de la fundamentación misma.

Otra idea que podemos retomar a la luz del paradigma no cognitivista es el principio de universalizabilidad. En Gewirth, como vimos, este principio determina el reconocimiento de otros agentes y la consecuente atribución de derechos, pero en esta tradición no sólo constituye un paso formal como en el razonamiento de Gewirth. En su argumento, este paso es crucial porque significa la transición de los derechos prudenciales a los derechos morales, que luego da lugar al PCG, núcleo de su fundamentación. Pero desde una posición no cognitivista (en este punto tomamos la postura de Hare, 1999), el principio de universalizabilidad incluye elementos actitudinales o evaluativos, ya que prescribir una norma universalmente, necesariamente implica poder querer o desear, las preferencias de los demás agentes, lo cual puede llevarse a cabo a través de la imaginación, que juega un rol fundamental en su teoría. ${ }^{16}$ Esto puede ser relevante a nuestros fines ya que una de las características especialmente distintivas de los derechos humanos es su supuesto alcance universal. De modo que puede funcionar como criterio, por ejemplo, para determinar un dominio de intereses básicos que conciernen a todos los agentes, pero además se puede articular con el aspecto motivacional y evaluativo, que suele ser un déficit de la tradición racionalista.

Para finalizar, queremos destacar que una de las principales razones por las que elegimos este punto de vista es porque consideramos que puede brindar un enfoque
16. "A menos que quiera, ahora, que la tortura se detenga en la situación hipotética, no estaré pensando en la víctima de la tortura como siendo yo... A menos que se me represente completamente cómo sería para míhallarme en esa situación, no estaré en plena posesión de los hechos de la situación. El argumento puede continuar entonces. Se sigue que siestoy en plena posesión de los hechos de la situación (y por supuesto, si no lo estoy, se me puede culpar de ignorar los hechos), entonces no seré capaz de querer que la tortura continúe en el caso hipotético en el que yo soy la víctima... Si no puedo querer que se me trate de ese modo en esa situación, entonces no puedo querer universalmente que quienquiera que esté en esa situación sea tratado de ese modo." (Hare, 1999: 146 ss., cursivas del autor). 
ontológico-epistemológico más plausible en lo que refiere a los valores que se establecen como prioritarios, ya sea que refieran al bienestar físico o a valores de otro orden como la libertad y la autonomía. Una de las razones es que no se le asigna a éstos una explicación causal (al modo realista), y además explicar los derechos de esta manera nos permitirá, eventualmente, contar con las ventajas del no cognitivismo, por ejemplo, el vínculo de los juicios morales con la motivación, la negación de valores en términos absolutistas y los criterios de corrección que se derivan de ello, y esto además sin implicar necesariamente una posición relativista. Además esto no implica negar los elementos correspondientes a la naturaleza humana, en un sentido fáctico, ya que pueden tener lugar como propiedades que sobrevienen a los juicios evaluativos y que constituyen razones.

\section{Consideraciones finales}

Para finalizar, indicaremos las conclusiones en torno a las principales cuestiones que nos hemos propuesto resolver. En primer lugar, el análisis del concepto de agencia revela una acepción propiamente normativa que puede ser interpretada como continuadora de la tradición de los derechos naturales; sin embargo, a pesar de los elementos en común creemos que, desde un punto de vista que contempla los elementos propios de la ética contemporánea, podemos sostener que la lectura a la luz de la tradición naturalista opaca la amplitud del concepto de agencia en su rol fundacionista. En este sentido brindamos algunos lineamientos para pensar una alternativa que considere la normatividad desde un enfoque no cognitivista que asuma la dimensión fáctica de la naturaleza humana desde otra perspectiva. 


\section{Bibliografía}

" Ayer, A. J. (Ed.) (1981). El positivismo lógico (trad. Aldama, L.), México: Fondo de Cultura Económica.

» Beitz, C. (2009). The Idea of Human Rights. Oxford: University Press.

" Brink, D. (1989). Moral Realism and the Foundations of Ethics. Cambridge: University Press.

"Campbell, R. (2003). Moral Epistemology. En Zalta E. (Ed.). The Stanford Encyclopedia of Philosophy. Recuperado de https://plato.stanford.edu/archives/win2015/ entries/moral-epistemology/.

» Darwall, S., Gibbard, A. \& Railton, P. (1997), Toward Fin de siècle Ethics: Some Trends. En S. Darwall, A. Gibbard \& P. Railton (Eds.), Moral Discourse and Practice: Some Philosophical Approaches (pp. 179-198). Oxford: University Press.

» Frankfurt, H.G. (1971). Freedom of the Will and the Concept of a Person. The Journal of Philosophy, 68 (1), 5-20.

»Gewirth, A. (1984). The Epistemology of Human Rights. Social Philosophy and Policy, 1 (2), 1-24.

"Gewirth, A. (1996). The Community of Rights. Chicago: University Press.

" Gibbard, A. (1990). Wise Choices, Apt Feelings. Oxford: Clarendon Press.

» Gibbard, A. (1997). Wise Choices, Apt Feelings. En S. Darwall, A. Gibbard \& P. Railton (Eds.), Moral Discourse and Practice: Some Philosophical Approaches (pp. 179198). Oxford: University Press.

»Griffin, J. (2008). On Human Rights, Oxford: University Press.

» Hare, R.M. (1981). Moral Thinking: Its Levels, Method and Point, Oxford: Clarendon Press.

» Hare, R.M. (1999). Ordenando la ética: Una clasificación de las teorías éticas (trad. Vergés Gifra, J.). Barcelona: Ariel.

» Miller, A. (2013), Contemporary Metaethics: An Introduction, Cambridge: Polity Press.

" Nickel, J. (2003). Human Rights. En Zalta E. (Ed.). The Stanford Encyclopedia of Philosophy. Recuperado de https://plato.stanford.edu/archives/spr2017/entries/ rights-human/.

» Rabossi, E. (1989). El fenómeno de los derechos humanos y la posibilidad de un Nuevo paradigma teórico. Revista del Centro de Estudios Constitucionales, 3, 323-343.

» Rorty, R. (1993). Human Rights, Rationality and Sentimentality. En S. Shute \& S. Hurley (Eds.), On Human Rights: The Oxford Amnisty Lectures 1993 (pp. 111-134). New York: Basic Books.

» Taylor, C. (1977). What Is Human Agency?. En T. Mischel (Ed.), The Self: Psychological and Philosophical Issues (pp. 103-135). Oxford: Blackwell.

» Walzer, M. (1994). Thick and Thin: Moral Argument at Home and Abroad. Notre Dame: University Press.

"Williams, B. (2006). Human Rights and Relativism. En G. Hawthorn (Ed.), In the Beggining Was the Deed (pp. 62-74). New Jersey: Princeton University Press. 
